\title{
V.
}

\section{Untersuchungen über den Puls mit Hülfe des Marey'schen Splygmographen.}

\author{
Von Dr. Koschlak off in St. Petersburg.
}

(Hierzu Taf. V u. VI.)

\begin{abstract}
Im Jahre 1861 schlug mir Herr Professor Botkin vor, Untersuchungen über den Puls mit dem Marey'schen Spbygmographen anzustellen. Je grösser die Zahl der Beobachtungen, welche ich an Gesunden und Kranken anstellte, wurde, desto mebr überzeugte ich mich, dass zur genauen Erklärung aller Eigenthümlichkeiten des durch den Sphygmographen erhaltenen Pulses die Beobachtungen, ebenso wie die zu diesem Zwecke angestellten Experimente nicht ausreichten. Es schien mir nothwendig, genauere Versuche anzustellen, um eínerseits mit der Wirkung des Instruments bekannt zu werden, d. h. seine Vorzüge und Mängel zu ersehen, und um andererseits diejenigen Bedingungen-zu bestimmen, von welehen die Eigenthümlichkeiten des Pulses abhängen. Die Lösung der ersten Aufgabe würde uns von der Möglichkeit befreien, die Fehler des Instruments für nothwendige Eigenschaften des Pulses zu halten; mit der Lösung der zweiten aber würde es uns möglich, über den Charakter der Bedingungen, unter welchen der Puls entstanden war, zu urtheilen. Deswegen führten wir im Anfange des Mai 1863 mit Hülfe des Sphygmographen eine Reihe von hydraulischen Versuchen an elastischen Röhren und Arțerien aus.

Um die Wirkung des Instruments $\mathrm{zu}$ erlernen, ordneten wir die Versuche auf folgende Weise an: Die Pulswellen wurden durch den Druck einer Wassersäule von bestimmter Höhe hervorgebracht. $\mathrm{Zu}$ den verschiedenen Versuchen wurden Wassersäulen von verschiedener Höhe genommen: $\frac{1}{2}, 2$ und $3 \mathrm{Mm}$. Zu jeder Versuchs-
\end{abstract}




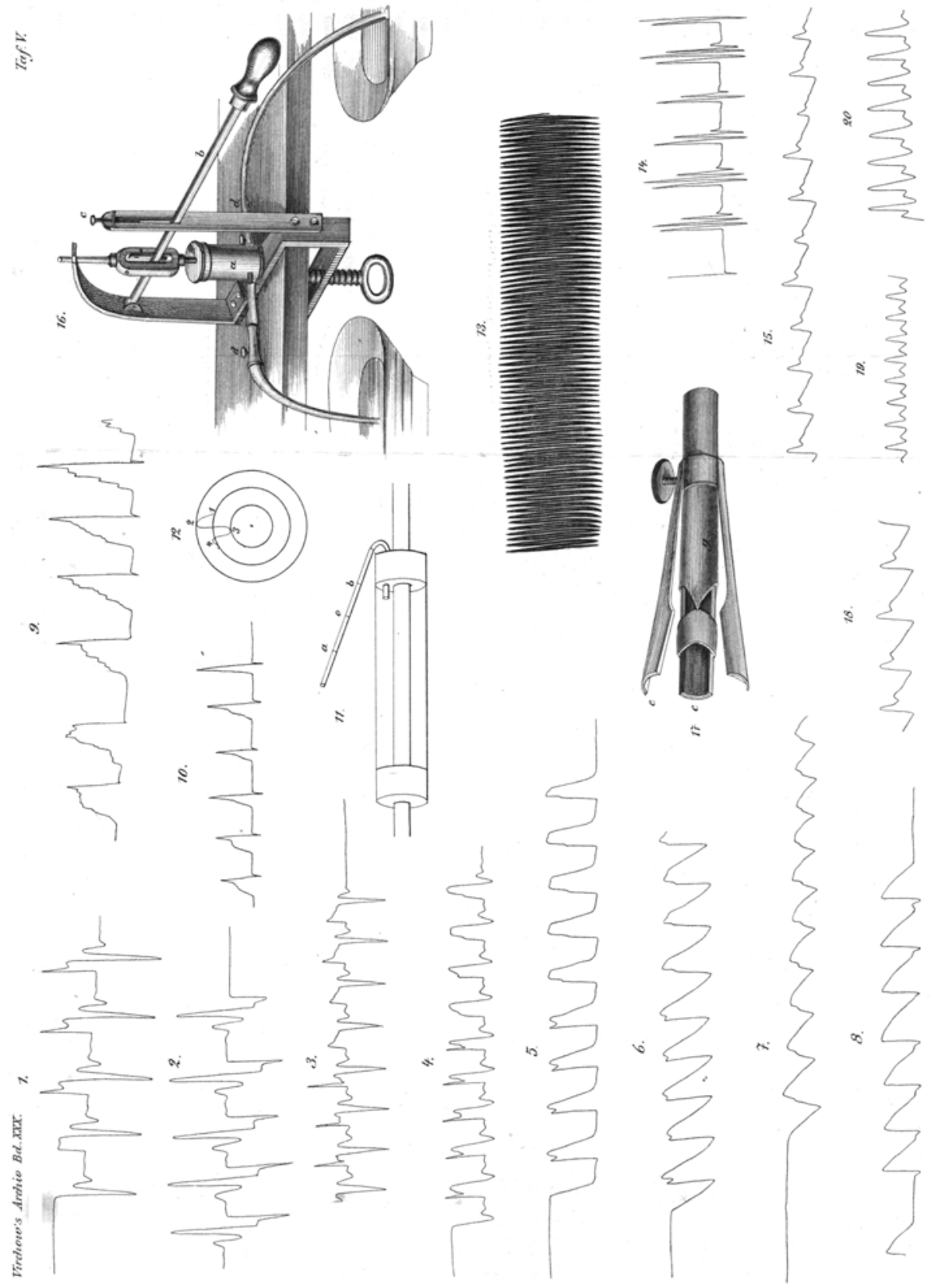



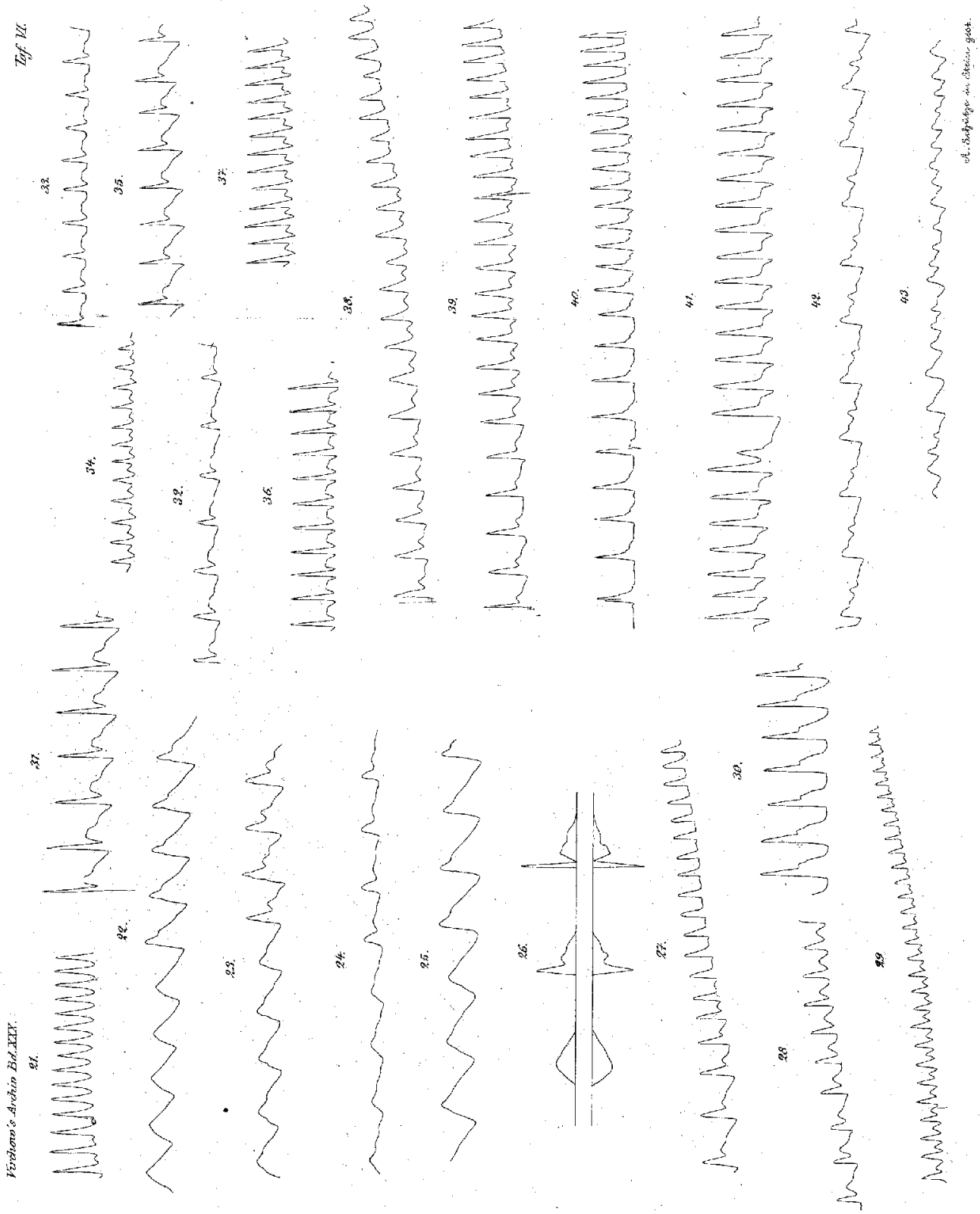
reibe nahmen wir fünf elastische Röhren von verschiedenem Durchmesser und verschiedener Elasticität. Der ganze Apparat bestand aus einer breiten (1,2 Cm. im Durchmesser haltenden) gläsernen Röhre, welche unten mit einem kupfernen Hahne versehen war und oben mit einem breiten, mit Wasser gefülten (damit beim Herausströmen der Flüssigkeit der Druck in kurzen Zeiträumen sich so wenig als möglich verändere) Gefässe in Verbindung stand. Auf das Ende des Hahnes wurde eine elastische Röhre gesetat und horizontal angeordnet; auf diese Röhre befestigten wir den Sphygmographen. Nachdem der ganze Apparat mit Wasser gefüllt war, öffnete und schloss ich den Hahn. Die Angaben des Sphygmographen wurden in jeder Versuchsreibe durch einen besondern manometrischen Apparat controlirt. Wir stellten die Experimente deswegen in dieser Form an, weil es uns dabei möglich wurde, die Grösse der Abweichung des Hebels von der Normalgrenze bei seinem Auf- und Niedersteigen zu bestimmen.

Um die Grösse des Steigens und Fallens des Hebels bëim Oeffnen und Schliessen des Hahnes zu bestimmen, bedienten wir uns zweier durch den Sphygmographen beim Oeffinen und Schliessen des Hahnes gezeichneter Linien. Wern man den Hahn öffnet, so dehnt das unter einem gewissen Drucke herausfliessende Wasser die Pöhre bị zu einem gewissen Grade aus, und diese Ausdehnung bleibt constant (wäbrend des kurzen Zeitraumes, der dazu gebraucht wird); in Folge der Ausdehnung der Röhre hebt sich der auf ihr befindliche Hebel empor und bleibt auf einer gewissen Höhe stehen. Wenn jetzt eine Metallscheibe mit einem darauf geklebten, berussten Papierstreifen vermittelst eines Uhrwerks am Hebel vorbeigeführt wird, so zieht bei geöffnetem Hahne der Hebel die Gleichgewichtslinie zweier entgegengesetzt wirkender Kräfte Schwere der Wassersäule und Elastjcität der Röhre - m. Nachdem ich den Hahn mehrmals geöffnet und geschlossen hatte, schloss ich ihn, ehe noch die ganze Scheibe ablief und erhielt so die Nullinie o, welche der Stellung des Hebels bei geschlossenem Hahne entsprach. Man kann jede von diesen Linien getrennt auf einer besonderen Scheibe erhalten, wie es Fig. 1 und 2 zeigt. Beide Abbildungen sind bei raschem Oeffnen und Schliessen des 
Hahnes von einer $4 \mathrm{Mm}$. im Durchmesser haltenden und $0,5 \mathrm{M}$. langen Röhre bei dem Drucke einer 1,5 M. hohen Wassersäule erhalten worden.

Diese Abbildungen betrachtend sieht man, dass der Hebel bei raschem Oeffnen des Hahnes sehr viel höher über die Linie $m$ steigt und bei raschem Schliessen. unter die Nulllinie o fällt. Was ist nun die Ursache dieser verstärkten Schwingungen des Hebels? Um diese Frage zu beantworten, führten wir die folgende Reihe von Versuchen aus: Es wurde eine Wassersäule von $\frac{1}{2}$ M. (um kleinere Pulswellen zu haben) genommen und das freie Ende der elastischen Röhre durch Einsetzen von Glasröhren von verschiedenem Durchmesser allmälig verengt, die Pulswelle aber wurde bald durch rasches, bald durch langsames Oeffnen und Schliessen des Hahnes hervorgebracht. Dabei erhielten wir die folgende Reihe von Abbildungen: Fig. 3, das Ende der Röhre ist nicht verengt. Fig. 4, das Ende der Röhre ist bis auf $2 \mathrm{Mm}$. verengt. Fig. 5, das Ende der Röbre ist bis auf $1 \mathrm{Mm}$. verengt. Fig: 6, das Ende der Röhre ist bis auf $\frac{1}{2} \mathrm{Mm}$. verèngt. Fig. 7, das Ende der Röhre ist durch ein Haarröhrchen von $0,2 \mathrm{Mm}$. verengt.

Dịe erste Hälfte einer jeden Abbildung ist bei raschem, die zweite bei langsamem Oeffnen und Schliessen des Habnes erhalten worden. Wenn wir die der Systole entsprechende Linie in jeder Abbildung betrachten, so sehen wir, dass das Steigen des Hebels über die Gleichgewichtslinie $m$ nur bei raschem Oeffnen des Hahnes statifindet, und es ist überdiess desto unbedeutender, je enger das Ausgangsende der Röhre ist, so dass bei starker Verengung der Hebel die Linie $\mathrm{m}$ nicht übersteigt. Es ist nicht schwer sich zu überzeugen, dass dieses Steigen über die Gleichgewichtslinie eine Folge der. Trägheit des Hebels ist. Bei raschem Oeffnen des Hahnes ist die Pulswelle sehr steil und theilt während inres Durchganges unter dem Hebel demselben im ersten Moment eine solche Geschwindigkeit mit, dass sein Steigen rascher als die Ausdehnung der Röhre erfolgt und in Folge der. Trägheit, weiter, als es der Röhrenausdehnung zukommt, fortgesetzt wird; darauf, theils in Folge seiner Schwere, theils in Folge der auf ihn drückenden kleinen Feder, fällt er wieder auf die Röhre zu- 
rück, bildet in ihr eine Vertiefung, wird auf's Neue emporgehoben und folgt endlich ihren Bewegungen.

Bei langsamem Oeffnen des Hahnes ist die Pulswelle minder steil und der durch sie im ersten Moment dem Hebel mitgetheilte Stoss weniger stark, daher sind auch die durch die Trägheit bedingten Schwingungen des Hebels kleiner, oder fehlen sogar vollkommen. Je nachdem der in Folge der Trägheit emporgestiegene Hebel auf die Röhre zurückfällt, ob im Momente ihrer Ausdehnung oder im Momente ihres Zusammenfallens, wird auch die Vertiefung bald auf der aufsteigenden Linie, bald auf dem Gipfel der Welle, bald auf der fallenden Linie abgebildet sein.

Da mit der Verengung des Röhrenendes sich die Steilheit der Welle ebenfalls vermindert, so ist es zu begreifen, woher in diesem Falle die Trägheit des Hebels ebenfalls vermindert wird oder sogar gänzlich fehlt.

Aus dem Gesagten folgt aber gar nicht, dass das Steigen des Hebels über die Gleichgewichtslinie immer das Resultat seiner Trägheit ist. Wenn das Wasser in eine elastische Röhre rasch und stossweise dringt, so erweitert sich die Röhre bei jedem Stosse bedeutender, als bei beständig geöffnetem Hahne, obgleich in beiden Fällen die drückende Wassersäule dieselbe bleibt. In diesem Falle werden die Gipfel der Pulswellen den wirklichen Grenzen der Röhreverweiterung entsprechen, wenn sie auch über der Gleichgewichtslinie stehen werden. Wir haben gesehen, dass Verengung des Röhrenendes die Trägheit des Hebels bedeutend vermindert oder sie sogar gänzlich vernichtet. Dieses Umstandes wollen wir uns bedienen, um die Schwingungen der Röhrenwände über die Grenze des Gleichgewichts hinaus zu zeigen. Nachdem wir das Röhrenende bis zu $\frac{1}{2} \mathrm{Mm}$. verengt und mebrere Pulse durch rasches Oeffnen des Hahnes hervorgebracht hatten, öffneten wir den Hahn auf längere Zeit, um die Gleichgewichtslinie zu ziehen, dann saben wir, dass die Gipfel der Pulse über der Linie $m$ standen (Fig. 8). Je höher die Wassersäule ist, desto höher wird der Pulsgipfel über der Linie $m$ stehen. Der Gipfel einer solchen Welle wird immer abgerundet, der Gipfel aber einer durch die Trägheit des Hebels verunstalteten Welle wird im Gegentheil zu- 
gespitzt sein. Die Eigenschaft des Instruments, bei raschen Stössen Trägheitsschwingungen zu beben, welche Marey für einen nützlichen Mangel hielt, der ein Mittel die Hypertrophie des linken Ventrikels zu diagnosticiren abgeben sollte, verunstaltet nur den Puls und trägt nichts zur Diagnose bei, denn der zugespitzte Pulsgipfel wird auch bei normalem Zustande des linken Ventrikels erhalten und fehlt oft bei dessen Hypertrophie. Es muss also dieser Mangel beseitigt werden, was auch gar nicht schwer ist; man muss nur die auf die Arterie aufgelegte Feder unmittelbar mit dem Hebel verbinden, was eben $\mathrm{Mach}$ in Wien gethan hat*).

Betrachten wir jetzt das Fallen des Hebels oder die diastolische Wellenlinie. Aus den früheren Abbildungen wissen wir, dass wenn das Ausgangsende der Röhre nicht verengt ist, der Hebel bedeutend unter die. Nullinie sinkt, dass mit dem Wachsen der Verengung des Röhrenendes das Sinken weniger tief wird, um endlich bei einer Verengung von $1 \mathrm{Mm}$. ganz zu verschwinden. Ausserdem erhebt. sich der Hebel, nachdem er schon einmal unter die Nulllinie gesunken ist, auf's Neue über dieselbe; sinkt wiederum u. s. f., d. h. er zeichnet eine zweite kleinere Welle, zuweilen auch eine dritte (Fig. 1, 2, 3, 4). Nach dem Verschluss des Habnes vollzieht folglich der Hebel bei der Nullinie allmälig kleiner werdende Schwingungen.

Was ist die Ursache dieser consecutiven Schwingungen des Hebels nach dem Verschlusse des Hahnes? Sollten sie durch wirkliche in der Röhre entstandene Schwingungen bedingt sein, oder sind es bloss Folgen der Sehwingungen der Feder des Instruments? Es ist allgemein bekannt, dass eine aus dem Gleichgewicht gebrachte und losgelassene elastische Scheibe eine Bewegung in entgegengesetzter Richtung über die Gleichgewichtslinie hinaus ausführt und nachher iminer kleiner werdende Schwingungen nach beiden Seiten hin vollzieht. Es ist möglich, dass die -aus ihrem Gleichgewicht durch die Pulswelle gebrachte Feder des Instruments nach dem Verschlusse des Hahnes der Bewegung der sich entleerenden Röhre folgend unter die ihrer früheren Lage entspre-

*) Die Arbeit Mach's ist uns zugekommen, als unsere Untersuchungen über das Instrument schon beendet waren. 
chende Linie sinkt und nachher auf der Röhre bei dieser Linie Schwingungen vollzieht, und je rascher nun sich die Röhre entleert, desto leichter die Schwingungen der Röhre erfolgen. Jetzt wollen wir diese Voraussetzung durch den folgenden Versuch controliren. Wenn wir den Sphygmographen auf eine mit Wasser gefüllte und an einem Ende offene Röhre befestigen, dann zwischen die Röhre und die Feder des Instruments einen Faden einführen, die Feder vermittelst des Fadens emporheben und loslassen, dann wird die Feder in Folge ihrer Elasticität sinken. Wenn wir dies mehrere Mal wiederholen, so erhalten wir die Abbildung Fig. 9.

Wenn wir den Hebel allein emporheben und fallen lassen, so wird eine der vorigen vollkommen gleiche Abbildung entstehen (Fig. 10).

Also führt die aus ihrem Gleichgewicht gebrachte und siç selbst überlassene Feder des Instruments die auf Fig. 1, 2, 3, 4 abgebildeten Schwingungen nicht aus: in beiden Fällen wurde nur die Vertiefung dureh das Fallen des Hebels bewirkt. Anders kann es auch nicht sein: die Feder sinkt in dem eben angegebenen Versuche nicht in einem freien Raume, sondern aut eine elastische Röhre, und je tiefer sie sinkt, desto grösser wird der Widerstand ron Seiten der elastischen Röhre sein. Es, ist augenscheinlich, dass unter diesen Bedingungen die Feder keine Schwingungen bei der Gleichgewichtsgrenze ausführen kann. Also müssen die consecutiven Schwingungen des Hebels bei der Nulllinie für den Ausdruck wirklicher Röhrenwandschwingungen angesehen werden. Von der Richtigkeit des Gesagten werden wir uns vollkommen überzeugen, wenn wir die Angaben des Sphygmographen durch einen anderen Apparat controliren. Wir nehmen eine an beiden Enden durch Pfropfen verschlossene breite gläserne Röhre, führen durch die in den Pfropfen angebrachten Oeffnungen eine elastische Röhre, durch eine zweite in einem der Pfropfen angebrachte Oeftnung führen wir das gebogene Ende einer manometrischen Röhre in die gläserne Röhre ein, füllen die gläsene, mithin auch die mañometrische Röhre mit Lakmustinctur und verbinden dann die elastische Röhre mit einer Wassersäule (Fig. 11), 
Jetzt bestimmen wir die Grenzlinien der Flüssigkeit bei geöfnetem und geschlossenem Hahn. Wenn wir den Hahn öffnen und das Wasser in die elastische Röbre fliessen lassen, so wird in Folge der Ausdehnung der elastischen Röhre ein Theil der Flüssigkeit aus der gläsernen in die manometrische Röhre treten und auf einer gewissen Höhe stehen bleiben. Wir notiren diese Höhe a und verschliessen den Hahn; die in der manometrischen Röhre befindliche Flüssigkeit wird sinken, weil die Wände der elastischen Röhre zusammenfallen und wird ebenfalls auf einer gewissen Höhe stehen bleiben. Bezeichnen wir diese Höhe durch 0. Jetzt wissen wir, bis zu weleher Höhe die Flüssigkeit im Manometer bei geöffnetem und geschlossenem Hahne gestiegen ist und fangen an, den Hahn zu öffnen und zu schliessen. Dabei sehen wir, dass beim Oeffnen des Hahnes die Flüssigkeit sich zuweilen nicht einmal bis zur Linie a erhebt, zuweilen aber dieselbe ubbersteigt und dass beim Schliessen sie entweder so weit wie sie sich über 0 erhoben, oder sogar noch tiefer bis $b$, unter Null sinkt, darauf sich auf's Neue über 0 erhebt, wiederum sinkt u. s. w., indem die Schwankungen immer kleiner werden. Dieses Experiment beweist auf's Augenscheinlichste, dass die durch die Flüssigkeit ausgedehnte elastische Röhre nach der Beseitigung der ausdehnenảen Kraft zusammenfällt und einen im Vergleich mit dem ursprünglichen viel kleineren Raum einnimmt*), darauf sich auf's Neue erweitert, wieder zusammenfällt, $d . h$. in eben solche Schwingungen, wie die stählerne Feder geräth. Dieses Ausdehnen und Zusammenfallen der Röhre kann dargestellt werden, wie in Fig. 12.

Die Zahl 1 entspricht dem Anfange der Ausdehnung, 2 dem Ende, 3 dem Ende des Zusammenfallens, 4 dem Ende der zweiten Ausdehnung. Wir müssen bemerken, dass wenn die zweite Erweiterung der Röbre erfolgt und die dritte, am Ende der Röhre Einsaugung der Flüssigkeit stattindet. Also entsprechen die zweite und dritte Welle dem negativen Drucke in der Röhre.

*) Dieses Zusammenfallen der Röhre wird noch durch die näb dem Verschlusse des Habnes in Folge der erhaltenen Geschwindigkeit sich weiter bewegende Fï̈ssigkeit begünstigt. 
Je mehr wir das Ausgangsende der Röhre verengen, desto unbedeutender wird das Sinken der Flüssigkeit unter Null, um endlich bei einer Verengung von $1 \mathrm{Mm}$. gänzlich zu verschwinden; bei solch einer Verengung steigt und fällt die Flüssigkeit im Manometer vollkommen gleichmässig und sinkt bei ihrem Rückgange nie unter 0 .

Diese Uebereinstimmung in den Angaben des Sphygmographen und des manometrischen Apparates beweist, dass die consecutiven Schwingungen des Hebels bei der Nullinie durch wirkliche in der Röhre entstandene Schwingungen bedingt sind, und dass die selbständigen Schwingungen der Feder keinen störenden Einfluss ausüben, denn die Schwingungen der Röbrenwände und die Schwingungen der Sphygmographfeder sind denselben Gesetzen der Elasticität unterworfen und werden, wenn die anderen Bèdingungen gleich sind, zusammentreffen.

Unter ungünstigen Verhältnissen aber entstehen die Schwingungen der Sphygmographfeder sogar da, wo sie in der Röhre fehlen. So etwas geschieht, wenn wir die Spannung der elastischen Feder bei unbedeutender Spannung der Röhrenwände vergrössern. Wenn wir eine Röhre von bedeutender Elasticität nehmen und den Sphygmographen auf sie inmer fester und fester schnüren, so erhalten wir bei einem gewissen Grade des Zusammenschnürens spontane Schwingungen der Feder, selbst bei immer geöffnetem Hahne. Dabei schwingt der Hebel ungemein rasch, so lange das Wasser herausfliesst und die auf einem Papierstreifen erhaltene Abbildung gleicht einem dichten Kamme (Fig. 13).

Wenn jetzt der Hahn geöffnet und geschlossen wird, so erhält man eine aus zwei, drei und mehr Schwingungen bestehende Pulswelle, je nachdem der Abstand zwischen dem Momente des Oeffnens und Schliessens grösser oder kleiner ist (Fig. 14).

Wenn wir den Hebel von der Feder abnebmen, so bören bei geringem Drucke der Wassersäule die spontanen Schwingungen der Feder auf, bei starkem aber führt die Feder auch allein diese Schwingungen aus. Man sieht daraus, dass der Hebel und die ihn andrückende kleine Feder die spontanen Schwingungen im In- 
strumente begünstigen. Bei Verengung des Röhrenendes verschwinden diese Schwingungen gänzlich.

Offenbar spielt die Feder in diesem Falle die Rolle einer elastischen Klappe. Angenommen, die Feder drückt die Röhre bis zum Verschwinden ihres Lumens zusammen. In dem vor der Feder liegenden Röhrentheile beginnt der Seitendruck stark anzuwachsen, in dem hinter der Feder liegenden Theile ist er gleich Null. In dem Maasse, wie das Wasser durch den geöffneten Habn in die Röhre dringt, wird der Druck in dem Eingangsende der Röhre so bedeutend anwachsen, dass die Feder die Röhre nicht mehr verschlossen halten kann und sich endlich in Folge des starken Wasserandranges emporhebt. Kaum hat sich aber die Feder emporgehoben und kaum ist ein Theil des Wássers aus dem Eingangs- in das Austrittsende geflossen, so vermindert sich plötżlich der Druck im Eingangsende und reicht dann nicht mehr aus, um die Feder emporzuhalten, sie fällt nun und verschliesst auf's Neue die Röhre. Das durch den geöffíneten Hahn zufliessende Wasser vergrössert wieder den Druck in dem Eingangsende, hebt wieder die Feder empor u. s. f. Da die Drackveränderungen in dem Eingangsende rasch erfolgen, so erfolgen die Schwingungen der Feder ebenso rasch. Wenn das Ausgangsende der Röhre verengt wird, so kann das Wasser aus dem Eingangsende, während die Feder emporgehoben ist, nicht rasch genug abfliessen, daher können in ihm keine raschen Druckveränderungen und folglich auch keine Schwingungen der Feder zu Stande kommen. Aus dem eben Gesagten sind auch die übrigen Einzelbeiten des Versuches zu begreifen.

Also sind die Fehler des Instruments sehr unbedeutend und bestehen nur in dem tieferen Sinken des Hebels nach dem Verschlusse des Hahnes. Die sponianen, unter besonderen Bedingungen sich bildenden Schwingungen der Feder werden schwerlich bei dem klinischen Gebrauche des Instrumentes entstehen; ich wenigstens habe so etwas nie beobachtet. Wenn man also die Trägheit des Hebels beseitigt, so wird das Instrument mit grösserer Genauigkeit arbeiten und das Sinken des Hebels wird unbedeu- 
tend, bloss bei raschen Veränderungen des Druckes in der Piöhre vergrössert.

Bei Betrachtung der systolischen Wellenlinie sagten wir gar nichts von der Einbiegung, welche in dieser Linie bei langsamem Einfliessen des Wassers in die Röhre entsteht, ob nun die Verlangsamung des Einfliessens durch ansehnliche Verengung des Röhrenendes (Fig. 7) oder durch langsamere Oeffnung des Hahnes bedingt wird. Diese Einbiegung hängt nïcht von der Trägheit des Hebels $a b$, sondern ist der wirkliche Ausdruck verschiedener Momente der Röhrenausdehnung. Es ist leicht, dieses mit Hülfe des manometrischen Apparates za beweisen. Bei dẽn oben angegebenen Bedingungen wird die Flüssigkeit in der manometrischen Röhre wäbrend der Systole in zwei Perioden steigen: nachdem sie eine gewisse Höhe erreicht hat, macht sie Halt und erhebt sich nach unbedeutendem Sinken auf's Neue, so lange die Systole dauert. Je kürzer die Systole ist, desto weniger ausgesprochen ist dieser Halt, welcher endlich gänzlich verschwindet. Also dehnt sich die Röhre, wenn das Wasser in sie langsam dringt, periodisch aus. Bisweilen hat die systolische Linie zwei Einbiegungen; hier dehnt sich also die Röhre in drei Perioden aus.

Um den Puls zu erlernen, wählten wir eine andere Form des Versuches. Wir suchten die mechanischen Bedingungen so ähnlich als möglich denen der normalen Blutcirculation zu machen. Anfangs stellten wir die Versuche in derjenigen form an, wie sie von Marey und Andern ausgeführt waren: wir brachten die Pulswellen, eine mit Klappen versehene bohle Kautschukkugel zusammendrückend, hervor. Aber diese Versuche erwiesen sich als ungenau, denn es war unmöglich, wie viel Mühe wir auch daran setzen mochten, das Zusammendrücken der Kugel mit gleichbleibender Kraft und Grösse auszuführen; unter diesen Umständen konnte aber weder die Dauer der Systole, noch die während des Zusammendrückens in die Röhre tretende Wassermenge sich beständig gleich bleiben. Diese beiden Umstände sind aber, wie wir später sehen werden, von grosser Wichtigkeit. Dabei entsprachen auch die Klappen, welche die Oeffnung entweder in Folge ihrer Schwere, oder in Folge ihrer Elasticität schlossen, nicht voll- 
kommen den Klappen des Herzens. Die Pulse, welche bei diesen Versuchen erhalten werden, sind niemals gleichmässig, was man aus der Abbildung Fig. 15, einer der gelungensten, die wir nur mit Hülfe dieses Apparates erhalten konnten, sehen kann.

Da wir bei solchen Versuchen keine genauen Resultate zu erhalten hofften, so richteten wir einen anderen Apparat ein, wit dessen Hülfe wir viel genauere Versuche anstellen konnten. In Fig. 16 ist dieser Apparat abgebildet.

Er besteht aus einer messingenen Pumpe a, welche durch den Hebel $b$ in Thätigkeit gesetzt wird. Vermittelst der Schraube c, auf welche sich der Hebel bei seinem Emporsteigen stemmt, wird sein Gang geregelt, d. h. es wird möglich, ihm einen Schwung von beliebiger, jedoch sich immer gleichbleibender Grösse zu ertheilen; dadurch wird es möglich, in die Röhre eine beliebig grosse, immer jedoch bestimmte Flüssigkeitsmenge einzuspritzen. Indem wir auf den Hebel Gewichte hängen, können wir. ihm eine beständige Kraft, mit der die Pumpe beim Hervorbringen der Pulswellen wirken wird, ertheilen. Das Gewicht rasch gegen den Anfang oder das Ende des. Hebels rückend, können wir diese Kraft während des Versuches plötzlich vermindern oder vergrössern. Auf die aus der Pumpe tretenden messingenen Röhren $d d$ werden die Klappen e aufgesetzt. Diese Klappen sind nach dem Vorbilde der Aortenklappen eingerichtet. In Fig. 17 ist eine solche Klappe in natürlicher Grösse dargestellt. Die messingene Röhre f hat auf einem Ende drei bogenförmige Ausschnitte, gegen welche die Klappen $g \mathrm{~g} g$ gedrückt werden. Zwischen die Klappen und die bogenförmigen Ausschnitte werden viereckige Handschubstückchen gelegt und die Klappen nachher angedrückt. Jetzt bilden die zusammengelegten Klappen ein Röhrchen, dessen Durchmesser dem Durchmesser des mit Ausschnitten versehenen Röhrchens gleich ist; die erste Röhre bildet also eine Fortsetzung der letzten. Die zwischen den Klappen und den Ausschnitten eingeklemmten Handschuhstïckchen bilden aber drei den Aortenklappen durchaus gleiche Klappen. Die herausstehenden Handschuhstiickchen werden beschnitten, und das Klappenröhrchen mit Seide bewickelt und mit Siegellack verklebt. Die so eingerichteten Klappen schliessen 
sehr fest, halten einen enormen Druck aus und verderben nicht, wenn man sie selbst mehrere Wochen lang beständig in Wasser hält. Ausserdem kann man in diesen Klappen eine beliebige Insufficienz herstellen.

Der so eingerichtete Apparat erlaubte uns, die Versuche auf's Verschiedenartigste zu variiren. Mit seiner Hulfe konnten wir die Kraft des Herzens in den weitesten Grenzen abändern; konnten die in die Röhre eingespritzte Wassermenge nach Belieben vergrössern oder vermindern, und waren hierbei immer überzeugt, dass in verschiedenen Versuchsreiben die Pulswellen unter denselben Bedingungen hervorgebracht waren.

Die Pulse, die vermittelst dieses Apparates hervorgebracht werden, sind durch Nichts vom menschlichen verschieden, und wir konnten ohne Mübe alle Pulsabänderungen ohne Ausnahme, wie normale, so auch pathologische, künstlich hervorbringen.

Alle ${ }_{2}$ Pulsformen, welche bei Kranken und Gesunden angetroffen werden, müssen in zwei Kategorien getheilt werden: 1) einfache (nicht dicrotische) und 2) doppelte (dicrotische) Pulse. Bei den ersten ist die diastolische Linie eben (ohne Einbiegungen) oder fast vollkommen eben; bei den anderen ist sie wellenförmig. Der dicrotische Puls zerfällt wiederum in mehrere Arten: in einen oberen Doppelpuls, wo der Anfang der diastolischen Welle über dem Anfange der systolischen steht; in einen mittleren, wo der Anfang der diastolíschen Welle auf gleichem Niveau mit dem Anfang der systolischen steht; und einen unteren Doppelschlag, wo der Anfang der diastolischen Welle niedriger, als der Anfang der systolischen steht (Fig. 18 bis 20).

Zu den dicrotischen Pulsen kann man den unteren nichtdicrotischen schlingenförmigen Puls rechnen (hier bildet die zweite diastolische Welle den Anfang der folgenden systolischen Linie und gibt dem Pulse das Ansehen einer Schlinge) Fig. 21.

Da die diastolische Linie im oberen Doppelpuls nicht eine, sondern mehrere Wellen bildet, so kann man ihn auch polyerotisch nennen; dann muss also der mittlere, eine diastolische Welle bildende Puls dicrotisch, und der untere, nur eine halbe diastolische Linie besitzende Puls halbdicrotisch genannt werden. 
Versuchen wir es jetzt mit Hülfe unseres Apparates die Bedingungen, unter welchen sich der dicrotische und der nichtdicrotische Puls bilden, zu bestimmen. $\mathrm{Zu}$ dem Versuche nehmen wir eine elastische $2 \mathrm{M}$. lange, $4 \mathrm{Mm}$. breite Röhre. Vermittelst der Schraube $\mathrm{c}$ wollen wir dem Hebel einen bestimmten Gang ertheilen; die Pulswellen werden in bestimmten Zeiträumen durch ein auf die Mitte des Hebels gehängtes Gewicht von $250 \mathrm{Gr}$. hervorgebracht. Während des Versuches wollen wir die Lage des auf der Röhre befestigten Sphygmographen in gar nichts ändern. Unter diesen Umständen.werden wir bald das der ausströmenden Flüssigkeit sich entgegenstellende Hinderniss, bald die Kraft des Herzens vergrössern, die Pulsfrequenz, die in die Röhre bei jeder Systole tretende flüssigkeitsmenge bleiben beständig dieselben.

Fangen wir unsere Versuche bei einer Verengung der Röhre von einem halben Millimeter an. Bei solch einer Verengung wird der Puls nichtdicrotisch sein. Wenn wir jetzt die Kraft des Herzens vergrössern, ohne in. Etwas die übrigen Bedingungen abzuändern, so erhalten wir einen dicrotischen Puls. Dieser Puls wird grösser, als der nichtdicrotische sein, die dicrotischen Wellen werden jedoch ebenso hoch, wie die nichtdicrotischen stehen. Die Neigung der sinkenden (diastolischen) Linie bleibt dieselbe, die steigende (systolische) Linie aber wird weniger schräg (mehr senkrecht). Um es augenscheinlicher $2 u$ machen; wollen wir auf derselben Scheibe die eine Hälfte der Pulse durch ein Gewicht von $250 \mathrm{Gr}$., die andere durch ein Gewicht von $500 \mathrm{Gr}$. zeichnen. Mit unserem Apparat ist es leicht, dieses anzustellen, man muss nur im Moment, wo der Hebel emporgehoben wird, das Gewicht von $250 \mathrm{Gr}$. von der Mitte auf das Ende des Hebels rücken. Die Abbildung ist bei einer Verengung von $0,5 \mathrm{Mm}$. erhalten worden (Fig. 22).

Verengen wir jetzt das Röhrenende. Mit jeder neuen Verengung verkleinert sich der Puls und der Doppelschlag; die steigende und sinkende Linie neigen sich mehr und mehr, und wir erhalten bei einer Verengung von $0,3 \mathrm{Mm}$. auf's Neue einen nichtdicrotischen Puls. Das Niveau einer ganzen Pulsreihe erhebt sich bei jeder Verengung; also wächst mit jeder Verengung đer Seiten- 
druck. Wenn wir jetzt wiederum die Herzkraft vergrössern, die übrigen Bedingungen aber dieselben bleiben, so erhalten wir auf's Neue einen dicrotischen Puls, auf derselben Höhe (der Seitendruck ändert sich nicht) und mit gleicher Neigung der sinkenden Linie, die steigende Linie aber wird, wie auch im vorigen Veisuche weniger schräg sein. Diese Veränderungen der Pulsform sind auf Fig. 23 dargestellt.

Wird die Röhre bis zu $0,1 \mathrm{Mm}$. verengt, so erhalten wir auf's Neue einen nichtdicrotischen Puls, vergrössern wir die Herzkraft, so wird er wieder dicrotisch (Fig. 24).

Diese Versuche kann man so lange fortsetzen, bis die in Folge der Verengung entstandene Spannung der Röhre so bedeutend wird, dass die Pulswellen kaum zu bemerken sind.

In diesen Versuchen erhielten wir aus nichtdicrotischen doppelte Pulse, indem wir die Kraft des Herzens vergrösserten. Der Seitendruck blieb dabei immer derselbe. Nichtdicrotische Pulse aber erhielten wir aus dicrotischen, indem wir das Hinderniss in dem Ausgangsende der Röhre vergrösserten. In diesem Falle wurde also der Seitendruck gesteigert. Man kann aber die eine Pulsform in die andere auch auf eine andere Weise überführen.

Stellen wir eine neue Versuchsreihe an. Wir beginnen bei einer Verengung des Röhrenendes von $0,1 \mathrm{Mm}$., einer Herzkraft von $2000 \mathrm{Gr}$. Die übrigen Bedingungen bleiben dieselben, wie in den früheren Versuchen. Jetzt vermindern wir abwechselnd bald die Herzkraft, bald das Hinderniss. Dabei werden wir dieselben Resultate, wie in der vorigen Versuchsreihe, nur in umgekehrter Ordnung erhalten. So erhalten wir Anfangs die zweite Hälfte der Fig. 24. Vermindern wir die Herzkraft um die Hälfte, so erhalten wir aus der $z$ weiten die erste Hälfte der Fig. 24. Erweitern wir das Ausgangsende der Röhre bis auf $0,3 \mathrm{Mm}$., so erhalten wir aus der ersten Hälfte der. Fig. 24 die zweite der Fig. 23. Wird die Herzkraft um die Hälfte vermindert, so erhalten wir aus der zweiten Hälfte der Fig. 23 die erste u. s. w. Dabei mussten wir, um aus dem dicrotischen einen nichtdicrotischen Puls zu erhalten, immer die Herzkraft, um aber aus dem nichtdicrotischen einen dicrotischen zu machen, das Hinderniss vermindern. Im ersten Falle wurde der 
stole ist, wollen wir versuchen zu erklären, woher und wie eine schnelle Systole den Doppelschlag hervorbringt?

Um diese Frage zu beantworten, werden wir den Zustand der Röhre während eines durch eine langsame Systole und während des durch eine rasche Systole hervorgebrachten Pulses betrachten. Zu diesem Zwecke wollen wir die Röhre im senkrechten Durchschnittsrisse darstellen. Dieser Durchschnittsriss kann ziemlich genau dargestellt werden. Da jeder Punkt der Röhrenwand sich während der Systole gleich weit ausdehnt und während der Diastole gleich weit zusammenfällt, so kennen wir, wenn uns die Form der Ausdehnung und des Zusammenfallens eines Punktes der Röhrenwand bekannt ist, die Form der Ausdehnung und des Zusammenfallens der Röhre in allen übrigen Punkten. Der durch den Sphygmographen gezeichnete Puls drückt die Ausdehnung und das Zusammenfallen der Röhre (Arterie) in einem Punkte aus, daher bedienen wir uns seiner, um den Durchschnittsriss der Röbre darzustellen.

Wir bringen drei pulsreihen durch drei Systolen von verschiedener Schnelle, bei in allem Uebrigen gleichen Bedingungen, hervor: für die erste Pulsreihe nehmen wir eine Systole (Gewicht) von $500 \mathrm{Gr}$, für die zweite die von $1000 \mathrm{Gr}$., für die dritte von 2000 Gr. Nachher nehmen wir den Durchschnittsriss der Röhre und legen auf jede Seite der Zeichnung in den entsprechenden. Punkten je einen Puls aus jeder Reihe auf, dann erhalten wir einen Durchschnittsriss der Röhre während dreier Pulse, welebe sich von einander nur durch die Schnelle der Systole unterscheiden (Fig. 26).

Aus der in Holzschnitt a wiedergegebenen Figur sieht man, dass die Röhre durch dieselbe einströmende Wassermenge desto mehr er-

a.

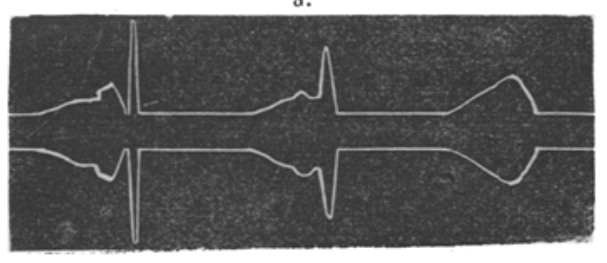
weitert wird, je rascher die Systole erfolgt. Ausserdem sieht man, dass nach Beendigung einer langsamen Systole die Röhre sich gleichmässig zusammen- . zieht, nach Beendigung 
Seitendruck nicht verändert, im zweiten aber verminderte er sich. Also können wir aus einem nichtdicrotischen einen doppelten Puls auf zweifache Weise erhalten: 1) wenn wir die Herzkraft, alle übrigen Bedingungen gleich gesetzt, vergrössern; 2) wenn wir das Hinderniss in dem Ausgangsende der Röhre vermindern, ohne die übrigen Bedingungen zu ändern. In beiden Fällen wird die Systole schneller zu Stande kommen.

Um aus einem doppelten einen nichtdicrotischen Puls zu erhalten, müssen wir entweder: 1) die Herzkraft vermindern, ohne die übrigen Bedingungen zu ändern, oder: 2) das Hinderniss im Ausgangsẹnde vergrössern, die übrigen Bedingungen gleichgesetzt. In beiden Fällen verlangsamen wir die Systole. Also ist eine schnelle Systole nothwendige Bedingung des Doppelschlages, eine langsame nothwendige Bedingung des nichtdicrotischen Pulses; der Seitendruck kann dabei beliebig gross sein.

Wenn wir die Angaben des Sphygmographen durch unsern manometrischen Apparat controliren, so finden wir eine vollständige Uebereinstimmung zwischen den ersteren und den Angaben des letzteren.

Es ist uns gelungen, die eben beschriebenen Versuche über das Hervorbringen der künstlichen Pulse auch an Leichen zu bestätigen. Wir vereinigten die Arteria subclavia mit unserem Apparat und spritzten in sie eine dem Blutserum entsprechende Eiweisslösung ein; solch eine Eiweisslösung gebrauchten wir, um das die Versuche sehr erschwerende Oedem der Extremität zu verhüten. Ungeachtet dieser Behutsamkeit aber stellte sich das Oedem dennoch ein. Aber im Anfange der Versuche konnten wir den dicrotischen Puls leicht in den nichtdicrotischen überführen, indem wir bloss die Kraft des Herzens veränderten. Auf der von der Radialarterie erhaltenen Abbildung (Fig. 25) ist die erste Hälfte durch ein Gewicht von $500 \mathrm{Gr}$., die zweite durch ein Gewicht von $1000 \mathrm{Gr}$. hervorgebracht.

Wir enthalten uns noch, aus den vorigen Versuchen weitere Schlüsse zu ziehen und fahren fort, die Erscheinungen des Doppelschlages zu untersuchen. Nachdem wir erfahren haben, dass eine nothwendige Bedingung seines Zustandekommens eine schnelle Sy- 
einer raschen ungleichmässig; sie ejweitert sich im letzteren Falle nach einer raschen und starken Contraction auf's Neue, zieht sich wieder zusammen u. s. w. und diese Contractionen und Erweiterungen werden desto grösser sein, je rascher die Systole war. Es ergibt sich aus diesem Umstande, dass die consecutiven Schwingungen der Röhre (Contraction und Erweiterung) unmittelbar von der Schnelligkeit und Grösse ihrer Erweilerung während der Systole abhängen. Wir wollen, um diese Abhängigkeit besser zu begreifen, das zwischen den Contractionen und Erweiterungen verschiedener Röhrentheile bestehende Verhältniss untersuchen. Der Anfang und das Ende der Röhre werden zu diesem Zwecke in manometrische Apparate eingeführt, die Pulse aber durch eine rasche Systole hervorgebracht. Um die Schwankungen der Flüssigkeit in beiden Apparaten gleichzeitig beobachten zu können, werden sie neben einander gestellt. Wir werden dabei sehen, dass die Flussigkeit in den Apparaten abwechselnd schwankt: wenn in dem näher zum Anfange der Röhre liegenden Manometer das Aufsteigen der Flüssigkeit schon beendigt ist, fängt es in dem mehr entfernten eben an; wenn die Flüssigkeit im ersteren fällt, geht im letzteren das Aufsteigen derselben vor sich; wenn im ersteren die Flüssigkeit zum zweiten Male steigt, so beginnt sie im letzteren zum ersten Male zu fallen u. s. w. Es erweitert sich also bei rascher Systole das eine Ende der Röhre, während das andere sich zusammenzieht, und umgekehrt.

Wenn wir jetzt die Pulse durch eine langsame Systole hervorbringen, so werden wir sehen, dass, sobald im ersten Manometer die Flüssigkeit bis zu $\frac{1}{2} 0$ der ganzen Höhe, auf welche sie sich während der Systole erhebt, gestiegen ist, im zweiten Manometer das Steigen derselben ebenfalls beginnt, wonach die Flüssigkeit in beiden Manometern parallel emporsteigt. Sogleich nach dem Beginn des Fallens der Flüssigkeit im ersten Manometer fängt sie auch im zweiten Manometer zu sinken an, worauf die Flüssigkeit in beiden Manometern parallel sinkt. Im ersten Moment sinkt die Flüssigkeit ziemlich rasch in beiden Manometern, später aber geschieht das Sinken langsamer, und wenn die Systole von langer Dauer war, so verlangsamt sich das Sinken der Flüssigkeit so be- 
deutend, dass sie sogar einen Halt macht. Den letzteren Fall werden wir später speciell untersuchen. Es erfolgt also bei langsamer Systole die Erweiterung und Contraction des Ein- und Ausgangsendes der Röhre fast gleichzeitig.

Daraus ist es leicht zu begreifen, woher sich die Röhre bei rascher Systole nicht gleichmässig, sondern, so zu sagen, sprungwéise contrahirt, während bei langsamer Systole die Contraction gleichmässig erfolgt.

Nach Beendigung einer raschen Systole wird die Differenz in der Spannung der Röhrenwände des Ein- und Ausgangsendes der Röhre eine sehr grosse, und zwar um so grössere sein, je rascher die Systole ist. Daher wird nach einer raschen Systole das Eingangsende der Röhre in Folge der grösseren Spannung seiner Wände bei gleichzeitig unbedeutender Spannung der Röhrenwände des Ausgangsendes sich sehr rasch contrahiren, dabei werden die Röhrenwände eine bedeutende Trägheit erhalten und werden sich in Folge dessen stärker, als es zur Herstellung des Gleichgewichts der Spannung in beiden Röhrenenden erforderlich ist, zusammenziehen. Es erfolgt auf diese Weise, nach Beendigung der Contraction des Röhrenendes, wiederum eine Störung des Gleichgewichts der Spannung; jetzt wird die in das Ausgangsende tretende Flüssigkeit die Wände desselben in eine grössere Spannung versetzen, während die Wände des Eingangsendes sich in einer geringeren Spannung befinden. Daher wird sich jetzl das Ausgangsende contrahiren, was die Erweiterung des Eingangsendes zur Folge hat, das Jetztere contrabirt sich auf's Neue und erweitert wiederum das Ausgangsende u. s. w. Diese abwechselnden Contractionen und Erweiterungen der Röhrenenden dauern so lange fort, bis sich das Gleichgewicht in der Spannung ihrer Wände herstellt.

Nach Beendigung einer langsamen Systole ist die Differenz in der Spannung der beiden Röhrenenden eine unbedeutende und sie wird überdies sogleich ausgeglichen durch den Abfluss der Flüssigkeit aus dem Eingangsende der Röhre in das Herz. Daher contrahirt sich auch die Röhre fast gleichmässig. Wenn das Gleichgewicht schon während der Systole hergestellt wird, so kann, 


\section{7}

wie wir später sehen werden, ein schwacher Dicrotismus Statt haben.

In wie weit aber sind die Resultate dieser Versuche zur Erklärung der Blutbewegung anzuwenden? Kann man auf ein ganzes Röhrensystem die Vorgänge, welche in einer Röhre beobachtet waren, übertragen? Bis jetzt hatte man gewöhnlich die Form des Pulses an einer Röhre studirt und, die Ergebnisse der Untersuchungen auf die Blutbewegung ubertragend, vieles durch Voraussetzungen ergănzt. Um zu erfahren, in wie weit diese Untersuchungen bei Erklärung der Blutbewegung anwendbar sind und um uns nicht in Muthmaassungen zu verlieren, wollen wir einige Versuche an Röhrensystemen anstellen.

$\mathrm{Zu}$ diesem Zwecke wollen wir das einfachste System wählen. Von der Hauptröhre leiten wir, 0,3 M. von ihrem Anfange entfernt, einen Zweig von 0,6 M. Länge ab. Auf das Eingangsende der Hauptröhre legen wir den Sphygmographen an und verengen das Ausgangsende bis auf $0,3 \mathrm{Mm}$. Die Hauptröbre soll die Aorta und die Arterien der unteren Extremitäten, der Seitenzweig aber die Arterien der oberen Extremitäten vorstellen. Jetzt unterbinden wir zuerst den Seitenzweig bei seinem Anfange und bringen dann doppelte Pulse hervor. Die dabei erhaltenen Pulse hahen die Form des Holzschnittes $b$.

b.

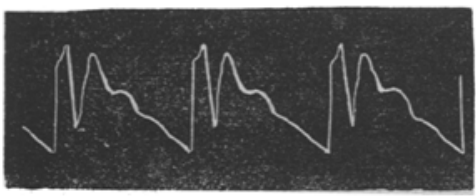

c.

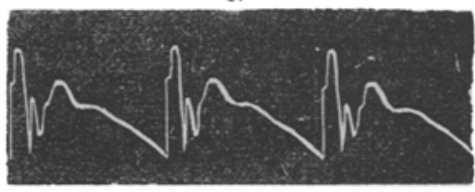

Wenn wir jetzt den Seitenzweig mit der Hauptröhre verbinden und die Ausgangsenden der beiden Röhren so verengen, dass das Hinderniss für das Ausströmen der Flüssigkeit ungefähr dasselbe wie im vorigen Versuche bleibt und doppelte Pulse hervorgebracht werden, so erhalten die Pulse die Form in Holzschnitt c.

Aus dieser Figur sehen wir, dass die frühere Pulsform durch das Hinzufügen eines Seitenzweiges einige Veränderung erlitten hat. Diese Veränderung besteht darin, dass im Zwischenraume 
zwischen der systolischen Welle und der ersten diastolischen noch ein e kleine Welle erschienen ist. Augenscheinlich ist. diese kleine Welle durch die Contraction des peripherischen Endes des Seiten$z$ weiges hervorgebracht worden. Nach dieser kleimen Welle folgten eben dieselben grossen Wellen und in derselben Reihenfolge, wie wir sie bei der Pulsation der nichtverzweigten Röhre erhalten haben. Untersuchen wir den Puls am Peripherieende des Seitenzweiges, so erhalten wir ganz dieselbe Form; auch hier werden die kleinen Wellen von ebenso grossen und in derselben Reihe nach einander folgenden Wellen begleitet, wie wir sie beim Anfangsende der grossen Röhre erhalten haben, nur erscheinen sie hier etwas später. Wenn wir den Puls längs des ganzen Vẹrlaufes der grossen Röhre untersuchen, so bemerken wir, dass die kleine Welle sich ziemlich

d.

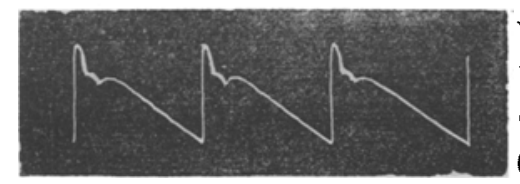
weit verbreitet, so dass auf dem von der Mitte der Röhre erhaltenen Pulse diese Welle noch deutlich zu unterscheiden ist (Holzschnitt d).

Aus den an verzweigten Röhren angestellten Versuchen müsste man schliessen, dass der doppelte Puls beim Menschen auf der diastolischen Linie $\mathrm{zwei}$ verschieden geformte Wellen haben sollte: 1) vordere, kleinere, welche von den Arterien des Kopfes und der. oberen Extremiläten herrühren, 2) grössere, die ersteren begleitende, die von den Arterien der unteren Extremitäten entstehen. Man findet auch wirklich solche Pulse, aber nicht immer; ausserdem 'sieht man bei diesen Pulsen nur eine einzige erste Welle und sehr oft $z$ wei Wellen der zweiten Ordnung. In den meisten Fällen jedoch

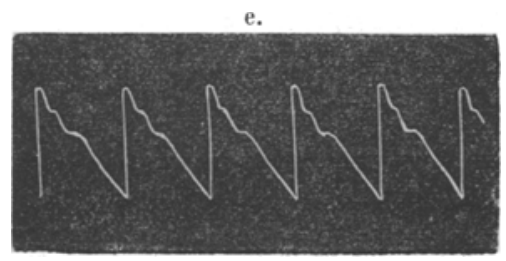
findet man entweder die kleine Welle gar nicht vor, oder sie wird durch eine leichte Wölbung des ersten Theiles der diastolischen Linie bezeichnet. An den auf Holzschnitt e abgebildeten Pulsen, die vom Menschen erhalten sind, sieht man deutlich die kleine und die sie begleitende grosse Welle; die übrigen sind. nicht so deutlich. 
Da man in den meisten Fällen die vordere Welle nicht vorfindet und da ihre An- oder Abwesenheit keinen grossen Einfluss auf die ubrigen Pulstheile hat, so 'sind die Ergebnisse der Pulsuntersuchungen an einfachen Röhren vollkommen anwendbar auf die Blutbewegung. Wenn wir unter dem Eingangsende die Aorta, unter dem Ausgangsende ihre Zweige uns vorstellen, so ist die Entstehung der doppelten Wellen im natürlichen Pulse leicht zu begreifen.

Wir haben gesehen, dass bei einer raschen Systole der Doppelschlag daher entsteht, dass bei ihrer Beendigung das Gleichgewicht in der Spannung der einzelnen Röhrentheile bedeutend gestört wird. Aber die Störung des Gleichgewichts kann auch bei einer langsamen Systole statthaben, und dann erhalten wir ebenfalls einen, wenn auch schwachen Dicrotismus.

Dieser Dicrotismus entsteht nach einer lange dauernden Systole. Wenn wir dem Hebel einen grossen Gang ertheilen und die Pulse durch kleine Gewichte hervorbringen, so wird der Hebel sehr langsam niedersinken. Wenn wir jetzt eine Röhre nehmen, deren Enden durch manometrische Apparate gehen, so werden wir Folgendes sehen: zuerst steigt die Flüssigkeit im ersten Manometer, darauf im zweiten und erhebt sich hernach in beiden parallel. Nachdem sie eine gewisse Höhe erreicht hat, bleibt sie zuerst im ersten, darauf im zweiten Manometer stehen und erhebt sich schon nicht mehr, abgleich die Systole fortdauert. Nach Beendigung der Systole beginnt die Flüssigkeit zuerst im ersten, darauf im zweiten Manometer zu sinken und fällt nachher in beiden Röhren parallel. Dieses Sinken geht im ersten Moment ziemlich rasch vor sich, darauf macht die Flüssigkeit Halt und sinkt nach diesem schon viel langsamer. Die unter solchen Bedingungen durch den Sphygmographen erhaltenen Pulse werden einen horizontalun Gipfel und eine manchmal von einer schwachen Welle begleitete Einbiegung auf der diastolischen Linie haben (Holzschnitt $f, g$ ).

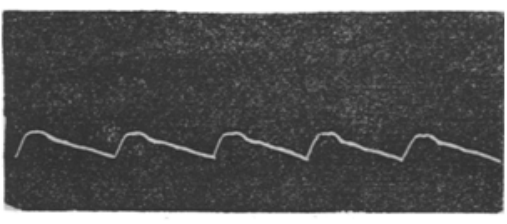

g.

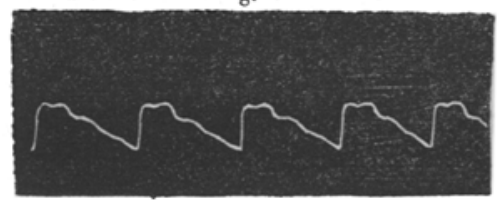


Die erste Figur ist bei einer Verengung von 0,1 Millim., die zweite von $0,3 \mathrm{Mm}$. erhalten. - Je mehr wir das Ausgangsende

h.

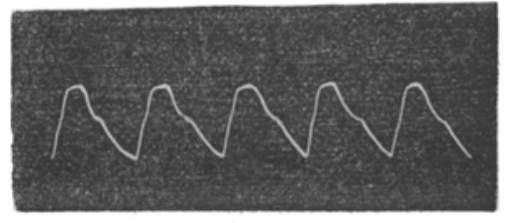

i.

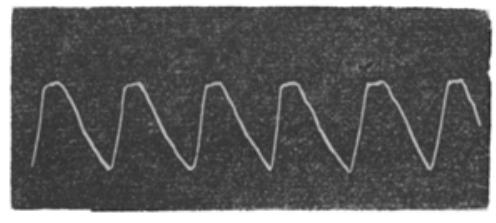
erweitern, desto weniger wird die Einbiegung auf der diastolischen Linie $\mathrm{zu}$ bemerken sein (Holzschn. h, das Ende der Röhre auf $0,5 \mathrm{Mm}$. verengt), so dass der Puls endlich vollständig nichtdicrotisch wird (Holzschn. i, das Ende der Röhre auf $1 \mathrm{Mm}$. verengt). Auch hier entsteht der Doppelschlag in Folge der Störung des Gleichgewichts in der Spannung.

Kurz vor Beendigung der Systole ist das Gleichgewicht in der Spannung aller Röhrentheile hergestellt; es wird dadurch bewiesen, dass die Röhre sich weder in ihrem Eingangs-, noch ihrem Ausgangsende mehr ausdebnt. Kaum ist die Systole beendigt, so strömt plötzlich eine bedeutende Flüssigkeitsmenge zurück zum Herzen, um die Klappen zu schliessen. Gleich nach dem Abströmen der Flüssigkeit fallen die Wände des Eingangsendes zusammen und werden weniger gespannt sein. Diese Verminderung der Spannung der Wände des Eingangsendes ist die Ursache des Dicrotismus. Dieser Dicrotismus beginnt also mit Verminderung der Spannung der Wände des Eingangsendes, nicht aber mit ihrer Vergrösserung, wie es bei rascher Systole der Fall ist. Beide aber werden durch dieselbe Ursache bedingt - rasche Störung des Gleichgewichts in der Spannung der einzelnen Röhrentheile.

Wenn rasche Systolen langsam auf einander folgen, so wird die Röhre während der Diastole Zeit haben, mehrere Schwingungen auszuführen, und der Puls wird dann ein polycrotischer, oder wenn von seinen diastolischen Wellen nur eine zu bemerken ist, ein oberer dicrotischer sein.

Wenn aber die Systolen rasch auf einander folgen, so hat die Röhre nur Zeit, zuweilen eine, zuweilen eine halbe Schwingung, zuweilen nur die aufsteigende Linie der diastolischen Welle aus- 
zuführen. Im ersten Falle wird der Puls ein dicrotischer oder doppelter mittlerer, im zweiten ein halbdicrotischer oder doppelter unterer, im dritten ein nichtaicrotischer unterer oder schlingenförmiger sein. Auf Fig. 27 sind diese Pulse abgebildet. Sie sind bei allmäliger Verschnellerung des Pulses erhalten worden. Das Steigen des Niveau's der Pulsreihen deutet auf Vergrösserung des Seitendruckes bei Verschnellerung der Pulsation. S. auch Fig. 38, 39 u. 40.

Dasselbe sieht man auch auf Fig. 28 u. 29, die zweite ist die Fortsetzung der ersten.

Ueber den Charakter der diastolischen Linie muss man Folgendes aussagen: je grösser das Hinderniss ist, desto langsamer erfolgen die Schwingungen der Röhrenwände während der Diastole, daher werden die diastolischen Wellen, alle übrigen Bedingungen gleichgesetzt, desto breiter sein, je grösser das Hinderniss in der Röhre ist.

Die Fig. 30 ist bei kleinerem, die Fig. 31 bei grösserem Hindernisse (Widerstande) erhalten worden.

Untersuchen wir jetzt die Bedeutung des nichtdicrotischen und des oberen dicrotischen Pulses. Nach Marey soll der erste auf eine Vergrösserung, der zweite auf eine Verkleinerung des Seitendruckes deuten. Wir haben aber gesehen, dass wie der dicrotische so auch der nichtdicrotische Puls bei demselben Seitendrucke entstehen können, wenn nur im ersten Falle die Systole rasch, im zweiten aber langsam erfolgt. Da es aber eine Menge Abstufungen in dem Seitendrucke geben kann, so gibt es such eine Menge dicrotischer und nichtdicrotischer, dem Drucke nach äquivalenter Pulse. Also kann der Dicrotismus oder Nichtdicrotismus des Pulses kein Criterium bei der Beurtheilung des Seitendruckes abgeben. Daher schlägt man zur Bestimmung des Seitendruckes vor, die Neigung der diastolischen Linie in Betracht zu nehmen: je schräger sie steht, desto grösser ist der Seitendruck*). Obgleich es bei

*) Da aber die Neigungsgrösse der diastolischen Linie von der Grösse des Hindernisses (Widerstandes) im Ausgangsende der Röhre abhängt, so werden wir der Wahrheit näher sein, wenn wir nach der Neigung der diastolischen Linie über die Grösse des Hindernisses, nicht aber über die Grösse des Seitendruckes urtbeilen. 
dem dicrotischen Pulse schwerer ist, als bei dem nichtdicrotischen, diese Neigung zu bestimmen, so ist es dennoch möglich, weil seine Wellen an der diastolischen, bei einer langsamen Systole sich bildenden Linie entstehen. Dabei können die Wellen selbst zur Beurtheilung des Hindernisses dienen; sie werden, wie wir es schon gesagt haben, desto breiter sein, je grösser das Hinderniss ist.

Wenn in Folge einer bedeutenden Pulsfrequenz der grösste Theil der diastolischen Linie verschwindet, so wird es unmöglich, nach ihrer Neigung über das Hinderniss zu urtheilen. In diesem Falle können wir unser Urtheil auf die Zahl der diastolischen Wellen, welche zwischen zwei Systolen stehen, gründen: bei derselben Pulsfrequenz werden breite, weniger zahlreich zwischen zwei Systolen stehende Wellen einem grösseren Hindernisse entsprechen, und umgekehrt enge und zahlreichere einem kleineren. Daher wird bei zwei gleich frequenten Pulsen der balbdicrotische einem grösseren Hindernisse entsprechen, als der dicrotische. Dabei werden die bei grösserem Hindernisse entstehenden Pulse kleiner, als die bei geringerem Hindernisse entstehenden sein.

Auf den Abbildungen Fig. 32-40 sind verschiedene Arten des dicrotischen Pulses bei verschiedenen Verengungen des Röhrenendes dargestellt. Bei jeder Verengung stellen wir drei verschiedene Stufen der Pulsfrequenz vor.

Das Röhrenende ist bis 0,2 Mm. verengt: Fig. 32 oberer Doppelpuls, Fig. 33 mittlerer Doppelpuls, Fig. 34 unterer Doppelpuls. Verengung von $0,3 \mathrm{Mm}$. : Fig. $35,36,37$.

Die Abhängigkeit der Breite der diastolischen Wellen von der Grösse der Verengung kann man noch besser bei allmäliger Verschnellerung des Pulses sehen. Verengung von 0,25 Mm.: Fig. 38 . Verengung von $0,5 \mathrm{Mm}$. : Fig. 39 . Verengung von $0,7 \mathrm{Mm}$.: Fig. 40.

Bei dem Hervorbringen der drei letzten Abbildungen blieb die Herzkraft und die eingespritzte Wassermenge beständig dieselbe.

Als wir diese Resultate mit den Resultaten der an Kranken und Gesunden angestellten Beobachtungen verglichen, überzeugten wir uns, dass sie vollkommen auf die Erscheinungen der Blutcircula- 
tion übertragen werden konnten. Die Ergebnisse dieser Beobachtungen theilen wir hier nur in allgemeinen Umrissen mit.

Der nichtdicrotische und der obere doppelte Puls sind wie dem Kranken, so auch dem gesunden Organismus eigen; der mittlere aber und der untere sind nur bei Kranken anzutreffen (eine Ausnahme bildet das Turnen). Der nichtdicrotische Puls deutet auch hier entweder auf ein vergrössertes Hinderniss in der Blutbewegung oder auf Verminderung der Herzkraft. Den Einfluss der Hindernisse auf die Form des Pulses kann man auch am Menschen graphisch demonstriren; man muss nur die Räumlichkeit des Arteriensystems verkleinern. Dieses kann man entweder durch Zusammenpressen der beiden Art. femorales, wobei die Zahl der Circulationswege bedeutend vermindert wird, oder durch Contraction der Arterien unter dem Einflusse der Kälte bewerkstelligen. In beiden Fällen wird der dicrotische Puls nichtdicrotisch. Noch leichter ist es, den doppelten Puls in den nichtdicrotischen überzuführen, wenn man die Venen der untersuchten Extremität durch eine Binde, die wie zum Blutlassen angelegt wird, zusammenpresst, wodurch wir ebenfalls die Hindernisse in der Circulation der untersucbten Extremität vergrössern. Dasselbe geschieht auch, wenn der Sphygmograph zu fest angelegt wird, wobei eine Menge Hautvenen unwegsam werden. Da man bei Subjecten, die einen vollen Arm haben, den Sphygmographen, um den Puls zu erhalten, sehr fest anlegen muss, so ist es begreiflich, woher man bei solchen Leuten beständig nichtdicrotische Pulse erhält. Dieser Umstand bewegte mich, die Schnüre, welche zur Befestigung des Sphygmographen dienen, durch einen besonderen Befestigungsapparat zu ersetzen.

Wenn in Herzkrankheiten die Circulationshindernisse nicht gut compensirt werden, so verschwindet oder vermindert sich bedeutend der Doppelschlag. In diesem Falle ist die Hauptursache des nichtdicrotischen Pulses die Schwächung der Herzthätigkeit, theils aber wird er auch durch die in Folge des Oedems und der Venenuberfüllung entstehende Vergrösserung der Hindernisse bedingt. Den Einfluss des Oedems kann man sehr gut bei den an Leichen angestellten Versuchen beobachten. Mit Vergrösserung des Oedems 
verkleinert sich der Doppelschlag und verschwindet endlich gänzlich. Wenn wir Einschnitte in der Hand der untersuchten Extremität anbringen, so erscheint der Doppelschlag auf's Neue. Bei der gesteigerten Herzthätigkeit während des Turnens vergrössert sich der Doppelschlag, ebenso wie der Seitendruck (in Folge der gesteigerten Pulsfrequenz).

Die Beobachtungen an Fieberkranken, einerlei was auch die Ursache des Fiebers sein mochte, zeigten, dass während des Fiebers jeder nichtdierotische Puls doppelt wird, der dicrotische aber einen höheren Grad des Doppelschlags erbält. Da dabei der Puls frequenter wird, so ist es begreiflich, woher während des Fiebers der obere dicrotische Puls in den mittleren und unteren ubergeht. Das Zusammentreffen dieser Umwandlungen des Doppelschlages mit Temperaturveränderungen ist durchaus zufällig und lange nicht beständig. Wenn die Temperatur z. B. $39^{\circ} \mathrm{C}$. hoch ist, so erhalten wir in einem Falle den oberen, in einem anderen den mittleren, in einem dritten den unteren Doppelschlag. Daher kann man den Sphygmographen nicht, wie es Wolf vorschlägt, zu Temperaturmessungen verwenden. Von der Richtigkeit des eben Ausgesagten wird es leicht sein sich zu überzeugen, wenn man den Puls bei Betrunkenen beobachtet. Bei Betrunkenen schlägt der Puls viel schneller und geht oft in den mittleren und selbst unteren Doppelschlag über, die Körpertemperatur aber ist oft um einen Grad unter der normalen.

Nachdem wir über den Puls im Allgemeinen gesprocben haben, wollen wir einige Worte über den Puls bei Herzkrankheiten sagen.

Wir hatten Gelegenheit, den Puls bei den verschiedensten Herzkrankheiten zu beobachten und sind zur Ueberzeugung gekommen, dass diese Pulse wenig Charakteristisches darbieten. Der Puls bei bedeutender Insufficienz der Aortenklappen während der vollkommenen Compensation ist gross und mit unbedeutendem Doppelschlag. Bei unbedeutender Insufficienz ist er vom normalen gar nicht zu unterscheiden. Bei einer bedeutenden Stenose der Aortenmündung ist der Puls ungemein klein und entspricht nicht der enormen Hypertrophie des Herzens; seiner Form nach ist er 
aber durch gar nichts vom normalen verschieden. Bei der Stenose der Aortenmündung, welche durch Insufficienz der Klappen complicirt wird, ist der Puls ebenfalls gar nicht charakteristisch, und der bei Marey abgebildete, von uns aber künstlich erhaltene Puls ist auch bei normalen mechanischen Bedingungen anzutreffen.

Der Puls bei Insufficienz der Valv. bicuspidalis ist gar nicht vom normalen $z u$ unterscheiden, und die bei Marey abgebildeten Pulse stehen in keiner Beziehung zur Insufficienz dieser Klappe, denn sie werden auch bei vollkommen gesunden Klappen angetroffen.

Weit bemerkenswerther sind die von der unregelmässigen Herzthätigkeit abhängenden Pulse, Diese Eigenthümlichkeiten werden sehr genau durch das Marey'sche Instrument gezeichnet. Am häufigsten trifft man sogenannte Herzpausen an. Diese Pausen sind selten wirkliche Pausen, sondern bestehen gewöhnlich aus einer eigentbümlichen Systole und Diastole. Die Systole zeichnet sich dadurch aus, dass sie 1) sehr klein ist, so dass die durch sie gebildete Pulswelle in der Radialarterie nicht empfunden wird, 2) um ein Viertel der ganzen Zeitdauer żwischen zwei Systolen fruher erfolgt, die Diastole aber um ein ganzes Viertel länger als gewöhnlich dauert.

Diese Form der Pause bleibt in allen Fällen dieselbe (Fig. 41).

Solche Pausen beobachtete ich am häufigsten bei durch verschiedene Ursachen bedingten Hypertrophien des linken Ventrikels; besonders häufig sind sie bei Arteriosclerosis; seltener bei Hypertrophien des rechten Ventrikels. Nicht selten jedoch trifft man sie auch da an, wo keine Hypertrophie der Ventrikel vorzufinden ist. Zuweilen werden diese Pausen von unangenehmen subjectiven Empfindungen begleitet. In anderen Fällen jedoch werden sie von den Kranken gar nicht bemerkt, entweder weil sich die Kranken an sie gewöhnen, oder weil diese Pausen von Anfang an keine subjectiven Empfindungen hervorrufen. Auf. Fig. 42 ist ein Puls abgebildet, den wir bei einem Kranken, welcher an Arteriosclerosis, parenchymatöser Nierenentzündung und Hypertrophie des linken Ventrikels litt, erhalten haben; hier wurde jeder Pulsschlag von einer Pause begleitet. 
Am leichtesten kommen diese Pulse beim Liegen auf der linken Seite und etwas auf dem Rücken zu Stande. Im Fieber werden sie seltner.

Die andere Eigenthümlichkeit des Pulses besteht darin, dass die einzelnen Pulsationen nicht gleichmässig auf einander folgen und nicht gleichkräftig sind (gemischter Puls) Fig. 43. Diese Form des Pulses trifft man ebendaselbst an, wo Herzpausen vorgefunden werden; es ist aber bemerkenswerth, dass sie nur Herzpausen begleiten. Bei Störungen der Compensation vergrössert sich diese Unregelmässigkeit, bei deren Wiederherstellung verkleinert sie sich, zuweilen bis zum völligen Verschwinden. Wahrscheinlich übt diese Form. der unregelmässigen Herzthätigkeit einen gewissen Einfluss auf die Compensationsstörungen aus. Beide Formen sind deutlicher bei geschwächter Herzthätigkeit ausgesprochen. Um jedoch die Bedeutung dieser Unregelmässigkeiten und ihren Zusammenhang mit der Hypertrophie des Herzens gehörig zu beurtheilen, sind weitere Beobachtungen nothwendig. Ausfübrlicher werde ich über meine am Pulse angestellten Versuche und Beobachtungen in einer besonderen Abhandlung sprechen.

\title{
VI. \\ Zur Entwickelung des Nervensystems.
}

Von Dr. V. Hensen in Kiel.

(Hierzu Taf. VIII.)

\begin{abstract}
Am 5. November vorigen Jahres machte ich dem hiesigen physiologischen Verein die unten folgende Mittheilung. Obgleich ich damals die Absicht hatte, nicht eher über diesen Gegenstand etwas zu publiciren, ehe inm das Studium geworden war, welches er zu verdienen scheint, ändere ich doch diesen Entschluss, weil die Arbeiten ausgezeichneter Fachgenossen mich dazu drängen;
\end{abstract}




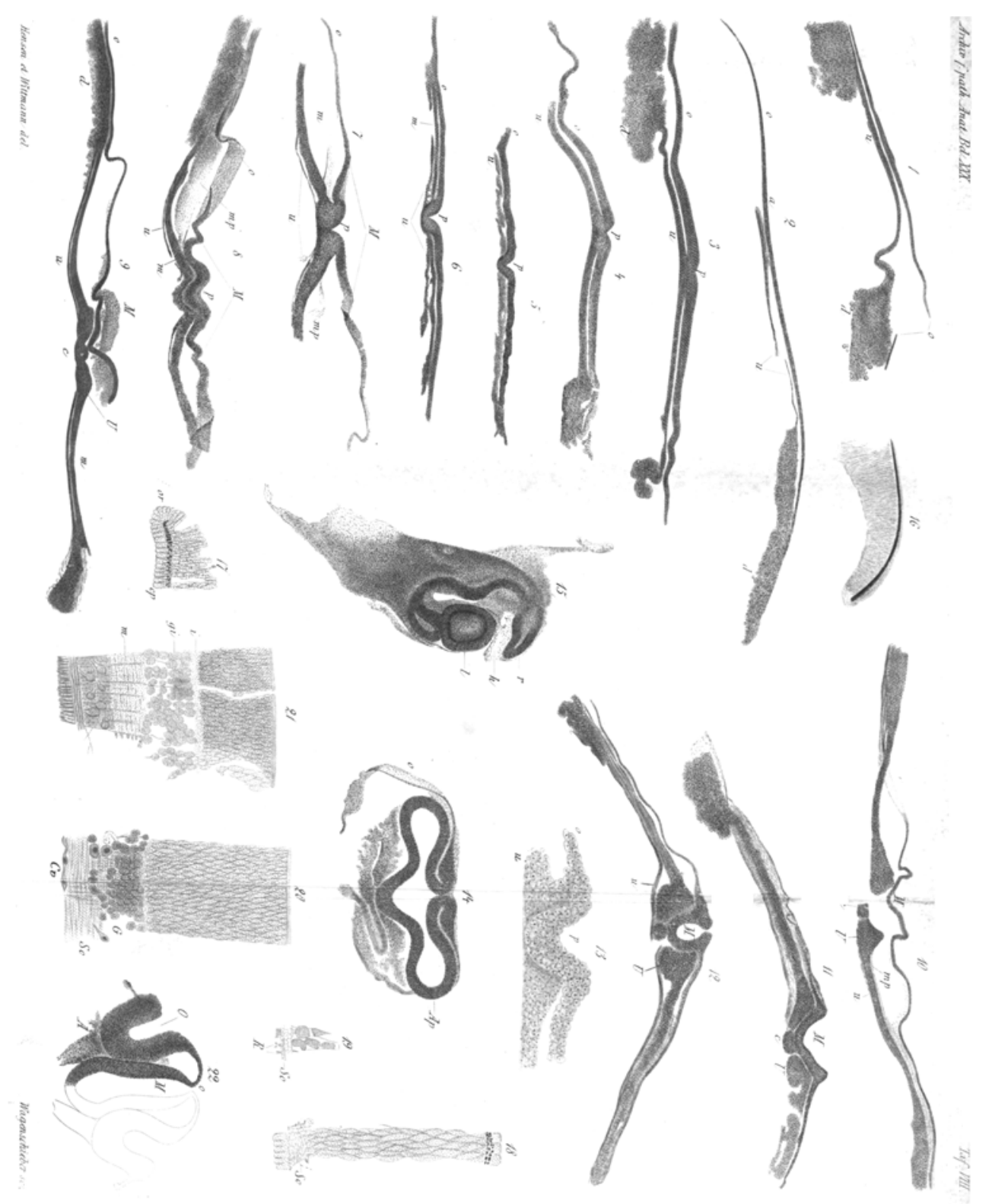

